\section{Case Reports in Dermatology}

\title{
Cholesterotic Fibrous Histiocytoma in a Patient with Metabolic Syndrome
}

\author{
Masatoshi Deguchi ${ }^{a, b} \quad$ Setsuya Aiba ${ }^{b}$ \\ ${ }^{a}$ Department of Dermatology, JR Sendai Hospital, Sendai, Japan; ${ }^{b}$ Department of \\ Dermatology, Tohoku University Graduate School of Medicine, Sendai, Japan
}

\section{Keywords}

Dermatofibroma - Macrophages - Mesenchymal tumors - Cholesterotic fibrous histiocytoma · Cholesterol cleft $\cdot$ Metabolic syndrome $\cdot$ Hyperlipidemia $\cdot$ Phagocytosis

\begin{abstract}
Among the many variants of dermatofibroma, dermatofibroma with cholesterol cleft (cholesterotic fibrous histiocytoma) is extremely rare. Here, we describe the case of a 50-year-old male patient with a cholesterotic fibrous histiocytoma on his left lower leg. He presented with a hyperkeratotic nodule $6 \mathrm{~mm}$ in diameter with a brown surface on the extensor surface of his left lower leg. The lesion had developed over the course of a few years without any tendency to heal. A skin biopsy performed on the tumor showed histopathological findings compatible with those of dermatofibroma. Interestingly, the lesion included many cholesterol clefts, as well as foamy histiocytes and multinucleated giant cells around them. He had had metabolic syndrome for years. To the best of our knowledge, this is the first report of a cholesterotic fibrous histiocytoma in a patient with metabolic syndrome. We conclude that the altered microenvironment caused by metabolic syndrome, as well as hyperlipoproteinemia itself, may play a role in the pathogenesis of this rare case.

(C) 2017 The Author(s)

Published by S. Karger AG, Basel
\end{abstract}




\section{Case Reports in Dermatology}

\section{Introduction}

Dermatofibroma has many variants, including cellular benign fibrous histiocytoma; angiomatoid-type, lipidized-variant, and granular cell dermatofibroma; epithelioid cell histiocytoma; atrophic dermatofibroma; and palisading fibrous histiocytoma. Among these variants, dermatofibroma with cholesterol cleft (cholesterotic fibrous histiocytoma) is one of the rarest forms. Histological findings of cholesterotic fibrous histiocytoma include cholesterol clefts within the center of the lesion, as well as variable degrees of invasion by foamy macrophages and giant cells around them. Furthermore, we could find only 3 definitive reports of cholesterotic fibrous histiocytoma associated with hyperlipoproteinemia. The first report was published by Hunt et al. [1] in 1990, followed by the reports by Yu et al. [2] and Takahashi et al. [3].

Recently, the concept of metabolic syndrome has been established. It was originally derived from the proposition by Reaven [4] named "syndrome X," where atherogenic risk factors combine with underlying insulin resistance. Others have developed this concept into metabolic syndrome, defined by a cluster of pathological conditions including abdominal obesity, dyslipidemia, hyperglycemia, and hypertension.

Here, we describe the first case of cholesterotic fibrous histiocytoma associated with metabolic syndrome.

\section{Case Report}

A 50-year-old male presented with a hyperkeratotic nodule $6 \mathrm{~mm}$ in diameter with a brown surface on the extensor surface of his left lower leg (Fig. 1). The lesion had developed over the course of a few years without any tendency to heal. He had been suffering from asteatotic dermatitis on his four extremities, and admitted that he had scratched his lower legs frequently before the tumor developed. He was treated for hyperlipidemia and hyperglycemia, in addition to control of abdominal obesity. The laboratory examinations from half a year before demonstrated serum total cholesterol levels ranging from 344 to $479 \mathrm{mg} / \mathrm{dL}$ (reference interval: $125-220 \mathrm{mg} / \mathrm{dL}$ ) with a low-density lipoprotein cholesterol level between 236 and $311 \mathrm{mg} / \mathrm{dL}$ (reference interval: 70-140 mg/dL), a triglyceride level of 1,650 $\mathrm{mg} / \mathrm{dL}$ at the maximum (reference interval: $45-150 \mathrm{mg} / \mathrm{dL}$ ), and hemoglobin $\mathrm{A}_{1 \mathrm{c}}$ ranging from 6.1 to $8.7 \%$ (reference interval: $4.3-5.8 \%$ ).

A skin biopsy performed on the tumor showed acanthosis and basal hypermelanosis of the epidermis. The poorly demarcated tumor mass was located in the dermis, separated by a clear zone from the epidermis, extending into the deep reticular area (Fig. 2a). The tumor was composed of an admixture of fibroblast-like spindle cells, rounded histiocytes, and capillaries. Spindle cells were arranged irregularly in the collagen strands. There were neither atypical cells nor bizarre cells in the lesion. Within the center of the lesion there were abundant cleft spaces compatible with cholesterol clefts (Fig. 2b), surrounded by foam cells and giant cells. Immunohistochemistry of the tumor revealed CD68-positive cells as well as numerous factor XIIIa-positive cells, in contrast to a negativity for CD34. Based on these findings, we diagnosed this lesion as cholesterotic fibrous histiocytoma.

Later, we performed total excision of this tumor, and the patient has remained free from recurrence for more than 5 years. 


\section{Case Reports in Dermatology}

\section{Discussion}

Our patient presented with dermatofibroma with many cholesterol clefts in the tumor mass, that is, cholesterotic fibrous histiocytoma. This case can be discriminated from tuberous xanthomas in that it had dermal cellular infiltrations by heterogeneous components including proliferative populations. They were composed of fibroblastic cells interspersed with collagen bundles and rounded histiocytic cells, in addition to foam cells and giant cell. Abundant factor XIIIa-positive cells in the tumor also supported the diagnosis of dermatofibroma in our case. Another differential diagnosis in this case includes lipidized fibrous histiocytoma. Although lipidized fibrous histiocytomas sometimes have cholesterol clefts, it is only a minor and infrequent finding [5] in their granulomatous lesions, whereas it is a predominant characteristic in our case; cholesterotic fibrous histiocytoma is defined as cholesterol deposition within the center of the lesion [2]. In addition, there was no hyalinized wiry collagen in the stroma of the specimen of this patient, which is known to be pathognomonic of lipidized fibrous histiocytoma [5].

The abdominal obesity, hyperglycemia, and hyperlipidemia (including hypertriglyceridemia) in our patient fulfilled the international criteria for metabolic syndrome $[6,7]$ as well as the Japanese standard [8]. To the best of our knowledge, this is the first case of cholesterotic fibrous histiocytoma in metabolic syndrome. The exact pathogenesis of cholesterol clefts remains to be elucidated. Yu et al. [2] proposed a hypothesis concerning the formation of cholesterol crystals in the lesional dermis; a possible local trauma may cause vascular leakage of lipoproteins followed by phagocytosis by dermal macrophages, resulting in such deposition. Actually, Takahashi et al. [3] demonstrated by observation with an electron microscope that in cholesterotic fibrous histiocytoma lesions, a cholesterol substance is incorporated in the histiocytes to form cholesterol crystals in the cytoplasm and lipid droplets in the lysosomes. Such a hypothesis seems to be most conceivable, since we can understand such a mechanism also by referring to xanthomas. As our patient had been suffering from itching of his lower legs due to asteatotic dermatitis, the scratching may have been responsible for minor injuries to the skin, leading to leakage of extremely high levels of serum cholesterol from the capillaries, as in reported cases of xanthoma $[9,10]$. The accumulated cholesterol, especially low-density lipoprotein, may be altered by the microenvironmental oxidative stress, making it susceptible to phagocytosis $[11,12]$. This speculation may be consistent with the common concept on the pathogenesis of atherosclerosis in the context of chronic inflammation including macrophage infiltration and oxidative stress [11-13]. Furthermore, the decreased level of plasma adiponectin found in metabolic syndrome patients [14] is well known to promote the uptake of oxidized low-density lipoprotein by macrophages, which may facilitate the formation of foam cells in the lesion. These microenvironmental conditions may combine to generate this rare variant of dermatofibroma. It is possible that with the increase in the number of cases with metabolic syndrome and hyperlipidemia in the general population, the number of patients with cholesterotic fibrous histiocytoma will increase as well. An analysis of case reports such as ours may enable us to predict the prognosis of metabolic syndrome and to elucidate the exact pathomechanism of cholesterol clefts themselves. 


\section{Statement of Ethics}

We confirm that the patient provided written informed consent to use his photographs for publication.

\section{Disclosure Statement}

The authors have nothing to disclose.

\section{References}

-1 Hunt SJ, Santa Cruz DJ, Miller CW: Cholesterotic fibrous histiocytoma. Arch Dermatol 1990;126:506508.

2 Yu M, Kim MS, Han TY, Lee JH, Son SJ: Cholesterotic fibrous histiocytoma. Am J Dermatopathol 2014;36:278-280.

- 3 Takahashi S, Hayama K, Inadomi T, Fujita H, Szuki H, Terui T: Electron microscopic observation of cholesterotic fibrous histiocytoma of the finger. J Dermatol 2015;42:923-924.

4 Reaven GM: Banting lecture 1988. Role of insulin resistance in human disease. Diabetes 1988;37:15951607.

5 Iwata J, Fletcher CDM: Lipidized fibrous histiocytoma. Am J Dermatopathol 2000;22:126-134.

-6 Expert Panel on Detection, Evaluation, and Treatment of High Blood Cholesterol in Adults: Executive summary of the third report of the National Cholesterol Education Program (NCEP) Expert Panel on Detection, Evaluation, and Treatment of High Blood Cholesterol in Adults (Adult Treatment Panel III). JAMA 2001;285:2486-2497.

7 Alberti KG, Zimmet P, Shaw J; IDF Epidemiology Task Force Consensus Group: The metabolic syndrome - a new worldwide definition. Lancet 2005;366:1059-1062.

8 Matsuzawa Y: Metabolic syndrome - definition and diagnostic criteria in Japan. J Atheroscler Thromb 2005;12:301.

$9 \quad$ Parker F: Xanthomas and hyperlipidemias. J Am Acad Dermatol 1986;122:1253-1257.

-10 Roederer G, Xhignesse M, Davignon J: Eruptive and tubero-eruptive xanthomas of the skin arising on sites of prior injury. Two case reports. JAMA 1988;260:1282-1283.

-11 Bergman R, Kasif Y, Aviram M, Maor I, Ullman Y, Gdal-On M, Friedman-Birnbaum R: Normolipidemic xanthelasma palpebrarum: lipid composition, cholesterol metabolism in monocyte-derived macrophages, and plasma lipid peroxidation. Acta Derm Venereol 1996;76:107-110.

-12 Gonzalez-Cuyar LF, Hunter B, Harris PL, Perry G, Smith MA, Castellani RJ: Cerebrotendinous xanthomatosis: case report with evidence of oxidative stress. Redox Rep 2007;12:119-124.

13 Ishibashi S, Goldstein JL, Brown MS, Herz J, Burns DK: Massive xanthomatosis and atherosclerosis in cholesterol-fed low density lipoprotein receptor-negative mice. J Clin Invest 1994;93:1885-1893.

14 Hulthe J, Hultén LM, Fagerberg B: Low adipocyte-derived plasma protein adiponectin concentrations are associated with the metabolic syndrome and small dense low-density lipoprotein particles: Atherosclerosis and Insulin Resistance Study. Metabolism 2003;52:1612-1614. 


\section{Case Reports in Dermatology}

Case Rep Dermatol 2017;9:136-140

(C) 2017 The Author(s). Published by S. Karger AG, Basel

Deguchi and Aiba: Cholesterotic Fibrous Histiocytoma in a Patient with Metabolic Syndrome

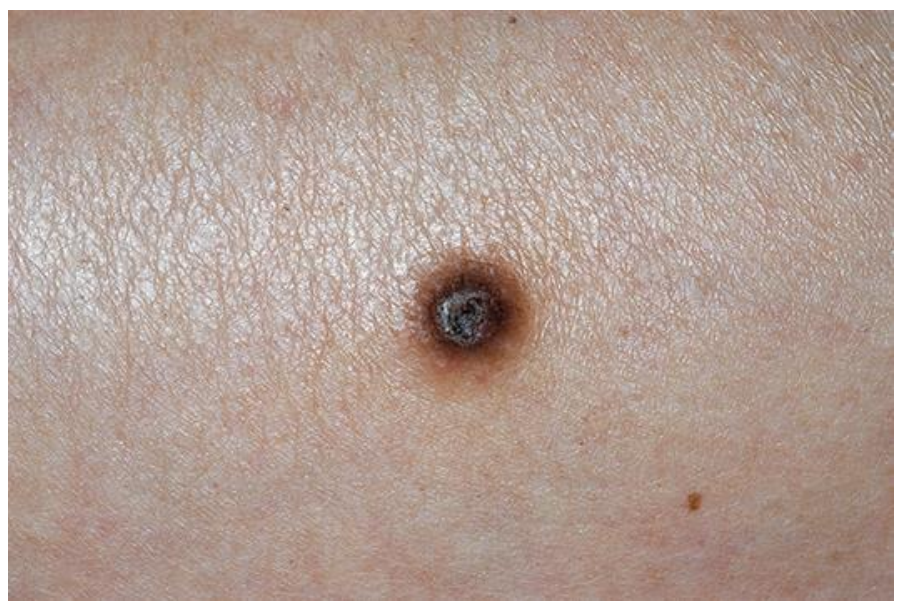

Fig. 1. Clinical appearance of dermatofibroma in the present patient.
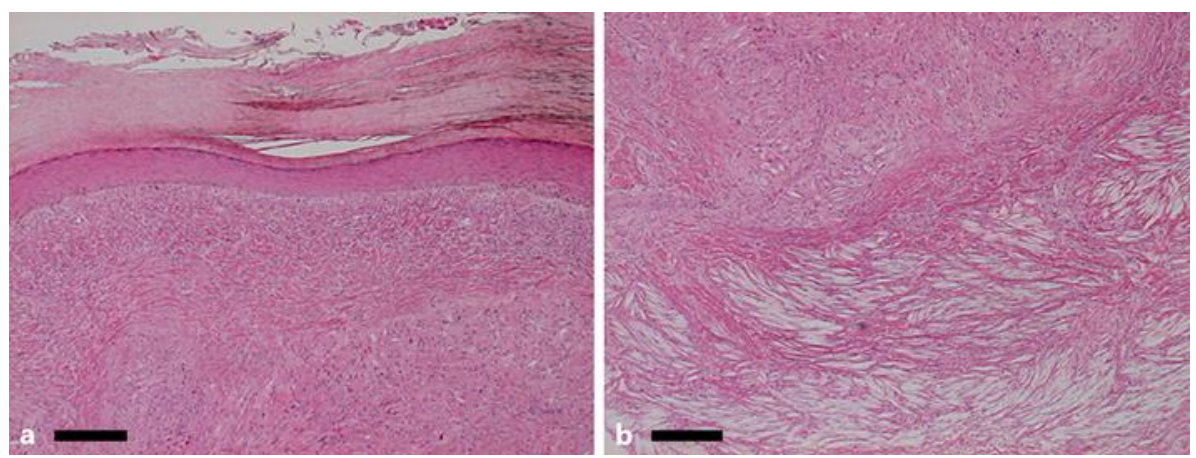

Fig. 2. a Histological findings from a biopsy specimen of the tumor. Scale bar, $200 \mu \mathrm{m}$. b Intratumoral layers of cholesterol clefts. Scale bar, $200 \mu \mathrm{m}$. 